Journal of Fundamental and Applied Sciences

ISSN 1112-9867

Available online at

http://www.jfas.info

\title{
CONTRIBUTION TO THE DEVELOPMENT OF A SIMULATION SOFTWARE PERFORMANCE AND SHARING RATIO IN LIQUID-LIQUID EXTRACTION
}

\author{
A. Hadj Seyd" and T. Lanez \\ Université $\mathrm{M}^{\text {ed }}$ Khider Biskra
}

Received: 22 October 2011 / Accepted: 11 December 2011 / Published online: 31 December 2011

\begin{abstract}
The present work is to develop software to predict the value yield and the distribution coefficient in the process of liquid-liquid extraction of components of a mixture, from mathematical models expressing these entities, based on equations equilibrium between liquid-liquid phases, and predict the conditions under which the extraction operation is favorable, unfavorable or impossible to realize, by studying the variation of the entities cited, based on the parameters influencing the extraction, which are: initial concentrations, rate of solvent and $\mathrm{pH}$, in the case of a simple extraction (extraction of neutral products) or when it is reactive (extraction of complex acids or bases) for one or more components.
\end{abstract}

The programming language used is "Delphi" which is a very powerful oriented object programming under Windows.

Key words: Liquid-liquid extraction, sharing, efficiency, partition coefficient, share ratio, solvent, solute, Delphi.

\section{INTRODUCTION}

L'estimation du rendement de l'extraction liquide-liquide d'un composé chimique, ainsi que la prévision de la valeur de son taux de partage entre les phases présentes dans un mélange, sont les facteurs les plus déterminants de l'opération d'extraction [1].

Author Correspondence, e-mail: seydtg@gmail.com

ICID: 1020755 
Ces entités varient en fonction de plusieurs paramètres notamment, les volumes des deux phases, la nature du milieu et des constituants, les concentrations des différents composés présents en solution ainsi que les coefficients de partage des substances à extraire $[2,3]$.

Afin de modéliser et d'optimiser le processus d'extraction, la conception par ordinateur de tels procédés requiert un modèle physico-chimique pour les équilibres liquide-liquide d'un système multi-constituants. Cette thématique a été largement abordée par plusieurs chercheurs et différents solvants et substances ont été étudiés [4-6].

L'objectif de notre travail est l'élaboration d'un logiciel permettant de prédire le rendement et le taux de partage d'extraction liquide-liquide à partir de modèles exprimant ces entités [7] et d'étudier l'influence des différents paramètres gouvernant le processus d'extraction, afin de prévoir dans quelles conditions cette opération est favorable, défavorable ou impossible, dans le cas d'une extraction simple (extraction de produits neutres) ou lorsque celle-ci est réactive (extraction de complexes, acides ou bases), pour un ou plusieurs constituants [8-10].

\section{MATERIELS ET METHODES}

Le logiciel a été compilé grâce à l'environnement de programmation DELPHI. La version utilisée est la septième version Française-Entreprise de DELPHI-2001 de la société Borland Software Corporation. L'environnement de développement DELPHI est considéré comme un très bon gestionnaire de base de données et un outil très puissant de programmation sous WINDOWS [11-12].

\section{RESULTATS ET DISCUSSION}

\subsection{Présentation du logiciel EXTRACT}

Le logiciel EXTRACT a été conçu pour faciliter les calculs de certains paramètres relatifs à une extraction liquide-liquide, notamment l'estimation des rendements et des taux de partage des constituants à extraire par des calculs basés sur la simulation informatique, et de prévoir dans quelles conditions l'opération d'extraction de ces constituants est favorable, défavorable ou impossible, toutefois en étudiant la variation de ces entités en fonction des paramètres les plus influençant sur l'extraction, à savoir, concentrations initiales, taux de solvant et concentration en ligands pour les complexes 
( $\mathrm{pH}$ pour les constituants acido-basiques). Le logiciel permet même de visualiser les variations de ces entités en les schématisant à l'aide d'une représentation graphique.

Le logiciel est valable pour une extraction d'un ou de plusieurs constituants, dans le cas d'une extraction simple (extraction de produits neutres) ou lorsque celle-ci est réactive (extraction de complexes, acides, bases ou ampholytes). Les différents cas aux nombre de 45 et dont les constituants du mélange pouvant aller jusqu'à cinq, peuvent être résolus à l'aide du logiciel EXTRACT.

Le logiciel EXTRACT compile aussi une base de donnée contenant plus de 2000 valeurs de coefficients de partage des substances chimiques les plus utilisées en laboratoire, dans une gamme importante de solvants au nombre de 36, l'utilisateur peut facilement consulter et même accéder à cette base de données afin de modifier ou ajouter des nouvelles substances, autres valeurs des coefficients de partage, de nouveaux solvants ou d'autres informations concernant ces substances tel que : formule chimique, masse molaire, densité, températures d'ébullition ...etc.

\subsection{Installation du logiciel $E X T R A C T$}

Le logiciel EXTRACT peut être facilement installé sous Windows en cliquant deux fois sur l'icône Setup à partir de son dossier d'installation et en suivant les instructions qui s'affichent sur l'écran. L'icône de son application sous forme d'une ampoule à décanter se situe automatiquement dans le menu démarrer de l'ordinateur.

\subsection{Utilisation du logiciel EXTRACT}

\subsubsection{Accès au logiciel}

L'application est lancée depuis l'icône EXTRACT du programme, un écran d'accueil de l'application s'affiche (splash) pour quelques secondes puis la fiche du menu principal apparaît, elle ressemble à la suivante (figure 1). 


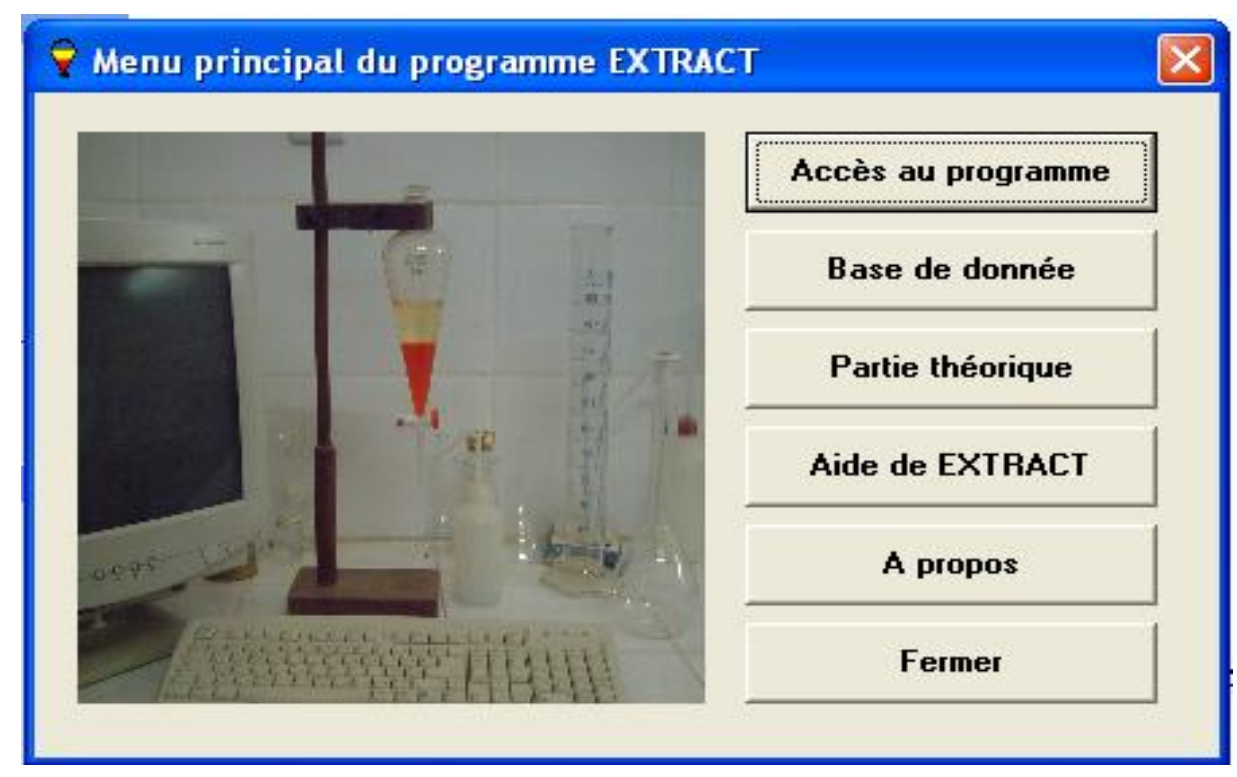

Fig.1. Menu principal du logiciel EXTRACT

L'accès au programme est possible par le biais du premier bouton en haut Accès au programme, une fiche intitulée Calcul des rendements et des taux de distribution est activée, elle est composée de trois feuilles de calcul distinctes:

- La première pour le cas d'une extraction simple

- La deuxième pour l'extraction réactive d'un seul composé

- Et la troisième pour l'extraction réactive d'un mélange

Donc selon la nature du composé extrait que l'utilisateur sélectionne la page de calcul correspondante.

\subsubsection{Cas d'une extraction simple}

Cette fiche est valable pour les composés neutres, autrement dit ceux qui ne réagissent plus dans l'eau, c'est en fait le cas de la plupart des composés organiques, les entités entrées sont:

La quantité initiale du composé à extraire exprimée en gramme ou en mole.

Les volumes de la phase organique et aqueuse exprimés en litre.

Le coefficient de partage du constituant entre les deux phases.

Et enfin le nombre d'extractions en cas d'application d'éventuelles extractions successives. 


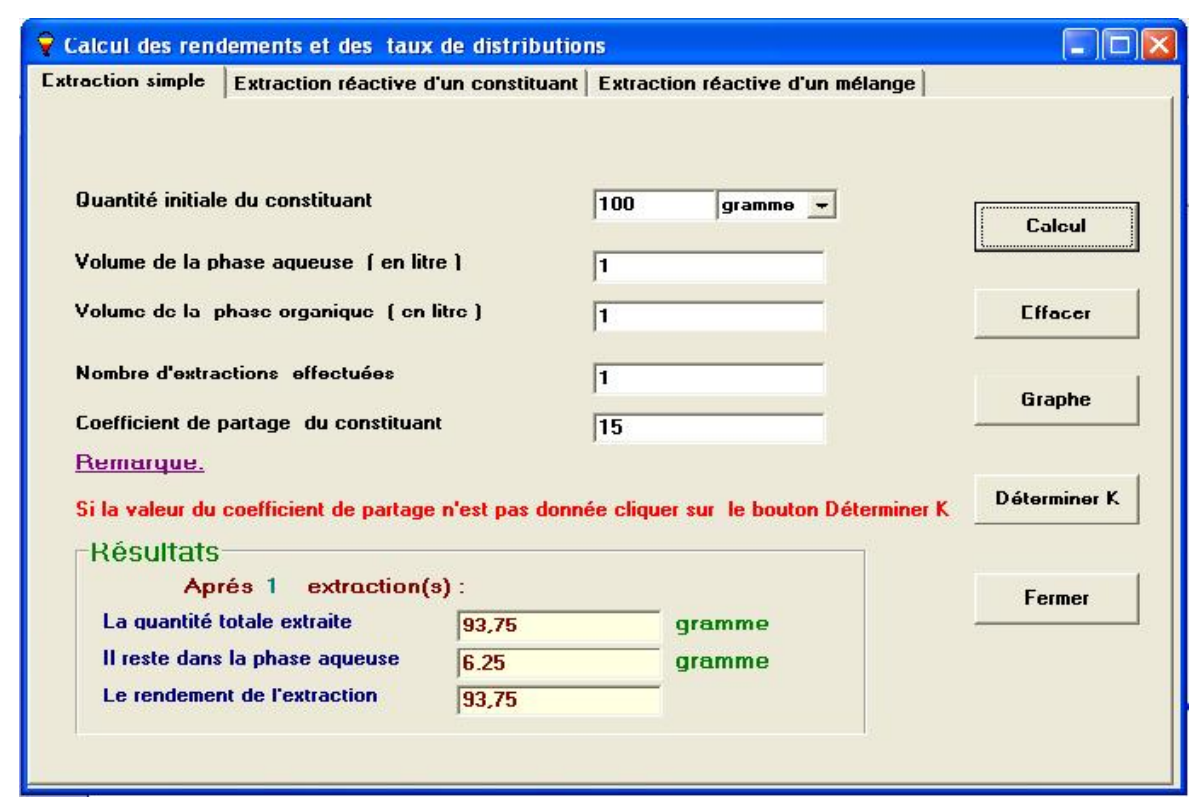

Fig.2. Feuille de calcul du rendement pour une extraction simple

Les résultats de calculs seront affichés dés que l'on appuie sur la touche Calcul, on pourra également remarquer que le rendement de l'opération n'est affecté par aucun paramètre, notamment $\mathrm{pH}$ ou $\mathrm{pL}$, et cela en accédant à la représentation graphique de $\mathrm{R}$ en fonction des paramètres suscité grâce à la touche Graphe. Le graphe $R=f(p H, p L .$. peut être visualiser en appuyant sur la touche Tracer, ce dernier devrait être une droite parallèle à l'axe des abscisses.

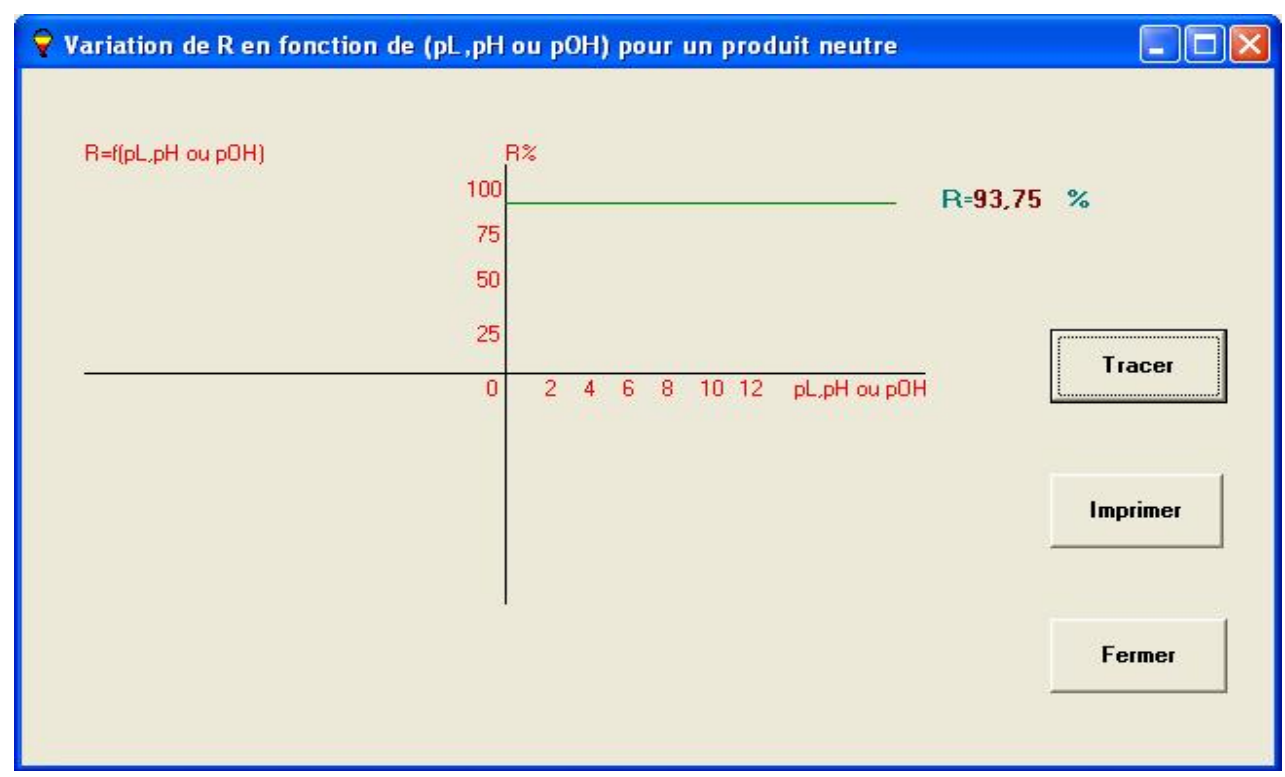

Fig.3. Représentation graphique du rendement pour une extraction simple 


\subsection{Cas d'une extraction réactive d'un seul constituant}

Une fois activée, la fiche de calcul dans le cas d'une extraction réactive d'un seul constituant ressemble à ce qui suit (Figure 4).

Il est primordial de préciser tout d'abord la nature du composé extrait, on trouve les possibilités suivantes : acide, base et complexe. De même que dans le cas précédent les entités entrées sont : les volumes des deux phases organiques et aqueuse exprimés en litre, la concentration initiale de la substance extraite en mol/l, s'agissant d'une extraction réactive c'est-à-dire la substance s'ionise dans la phase aqueuse, il faut donc préciser si cette dernière s'effectue en une ou plusieurs étapes et entrez le nombre de ligands libérés par le complexe (protons pour l'acide/base), selon la nature de cette substance, un message d'erreur est affiché en cas d'éventuelles erreurs.

Les calculs sont déclenchés en appuyant sur le bouton Calcul la valeur du pL ( $\mathrm{pH})$ de la substance en solution est déterminée automatiquement, les résultats des entités cherchées sont affichés à cette valeur de $\mathrm{pL}(\mathrm{pH})$, un message attire l'attention de l'utilisateur qu'il peut modifier cette valeur et refaire les calculs tout en indiquant la valeur optimale pour une extraction efficace.

Et enfin l'accès à une schématisation graphique des paramètres influençant sur l'extraction est effectué en appuyant sur le bouton Graphes les valeurs du rendement et des coefficients de distribution à différentes valeurs de $\mathrm{pL}(\mathrm{pH})$ sont affichés sous forme de tableau dés que la fiche est activée, les courbes de variations de ces entités seront tracées sur plan au biais des touches Graphe $\mathbf{R}$ ou Graphe LogKD.

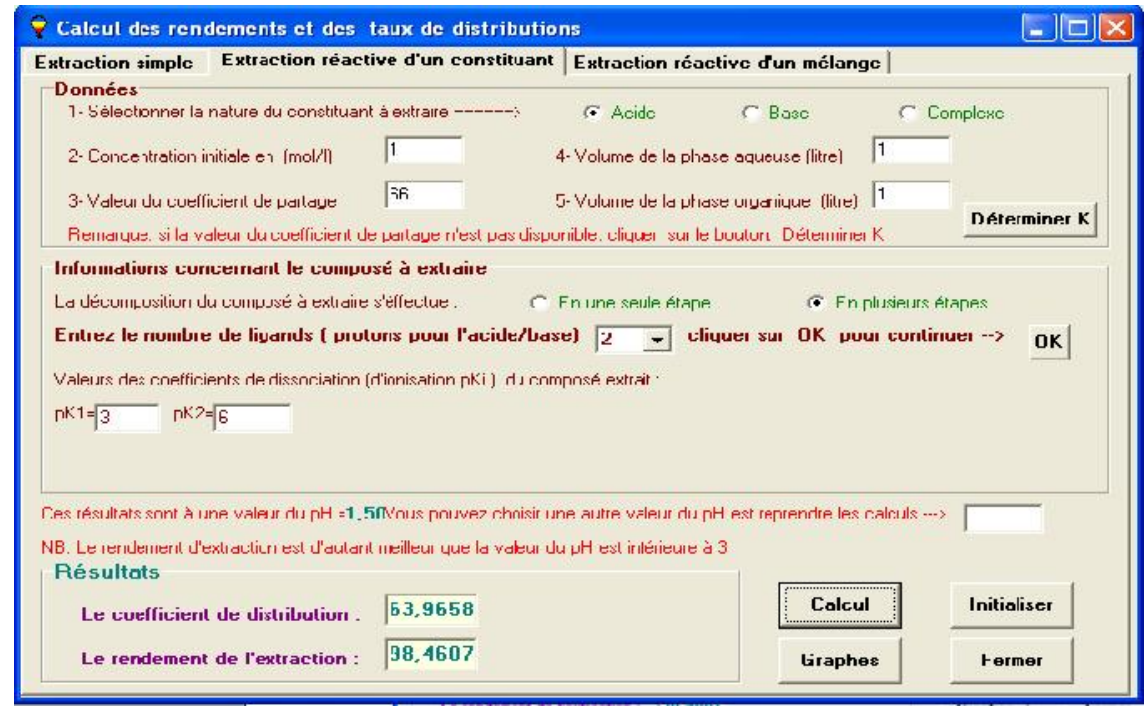

Fig.4. Feuille de calcul du rendement et du taux de partage pour une extraction réactive d'un seul constituant 


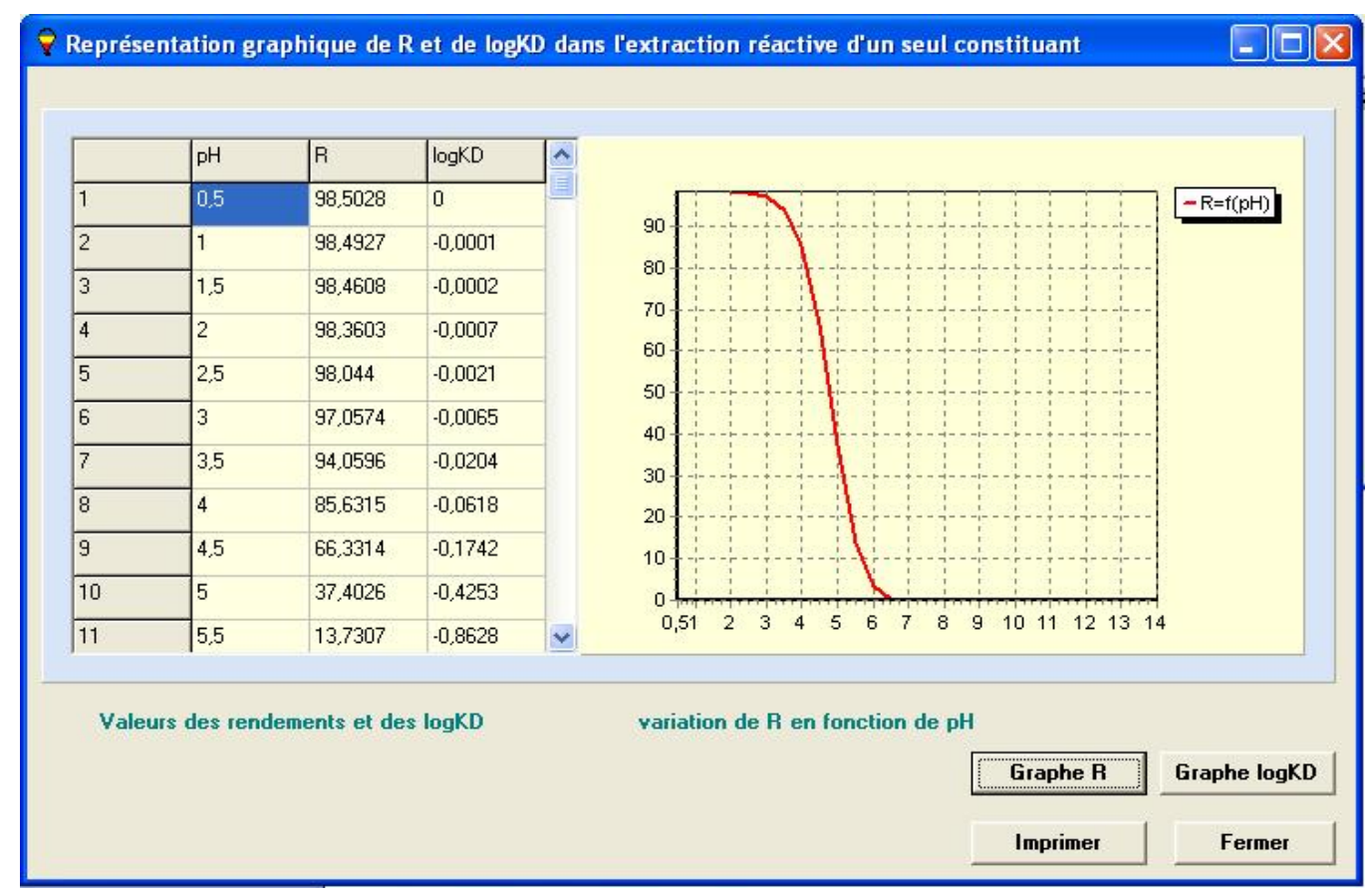

Fig.5. Variation du rendement pour une extraction réactive d'un constituant

\subsection{Cas d'une extraction réactive d'un mélange de constituants}

La figure 6 représente la fiche de calcul des rendements et des taux de partage pour le cas d'une extraction d'un mélange de constituants .Il faut tout d'abord sélectionner le mélange dans le menu contextuel, on a prévu 45 combinaisons de mélanges dont le nombre de constituants ne dépassant pas cinq, comportant des composés de même ou de nature différente pouvant être des acides, des bases, des complexes des produits neutres ou des mélanges de ceux-ci .Il faut aussi introduire les concentrations initiales, les constantes de partage et les constantes d'ionisation sous forme de $\mathrm{pKi}$ (constante de dissociation pour les complexes, d'acidité pour les acides). Dans ce cas, un message d'erreur s'affiche en cas d'introduction des valeurs erronées des pKi indiquant que le mélange est instable ou si la différence entre ces entités n'est pas suffisante pour que l'extraction ait lieu (la différence entre deux pKi doit être au moins égale à 2) .

Le $\mathrm{pL}$ ( ou $\mathrm{pH}$ ) est calculé automatiquement et les résultats des rendements et des coefficients de distributions de chaque produit seront affichés à cette valeur de $\mathrm{pL}(\mathrm{pH})$, un message indiquant à l'utilisateur la valeur optimale de cette entité qu'il faut choisir à fin d'extraire efficacement chaque produit du mélange, l'utilisateur peut réintroduire une nouvelle valeur et refaire ces calculs. 
La schématisation graphique des variations du rendement et des $\operatorname{LogKD}$ en fonction du $\mathrm{pL}(\mathrm{pH})$ est aussi possible en cliquant sur le bouton Graphes. La fiche de la représentation graphique (voir figure 7 ) permet l'affichage des valeurs des rendements et des taux de partage de chaque constituant du mélange en variant le $\mathrm{pH}(\mathrm{pL}$ pour les complexes), la représentation graphique de $\mathrm{R}$ ou de $\log \mathrm{KD}$ est possible en appuyant sur le bouton correspondant.

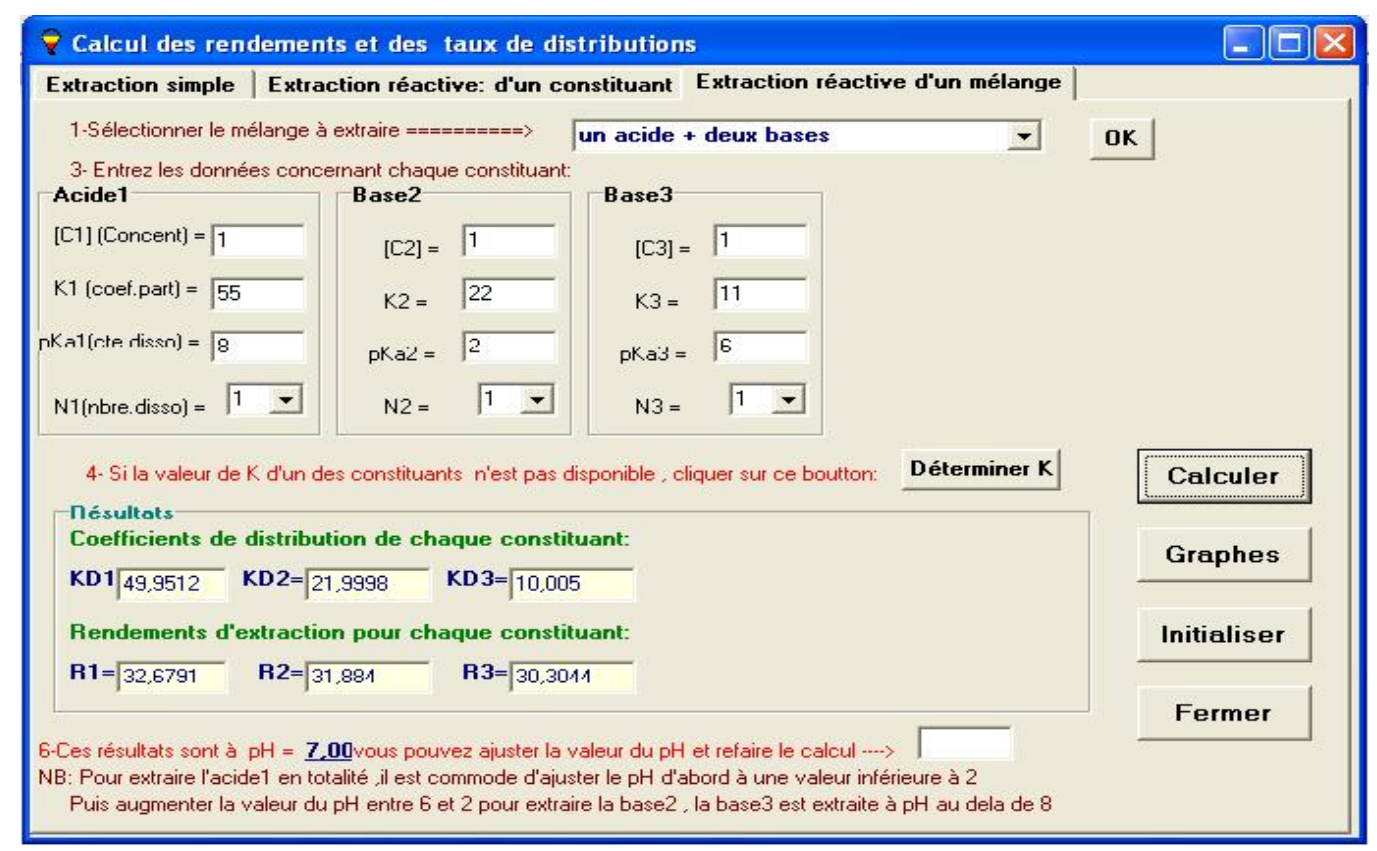

Fig.6. Fiche de calcul des rendements et des taux de partage pour le cas d'une extraction d'un mélange de constituants 


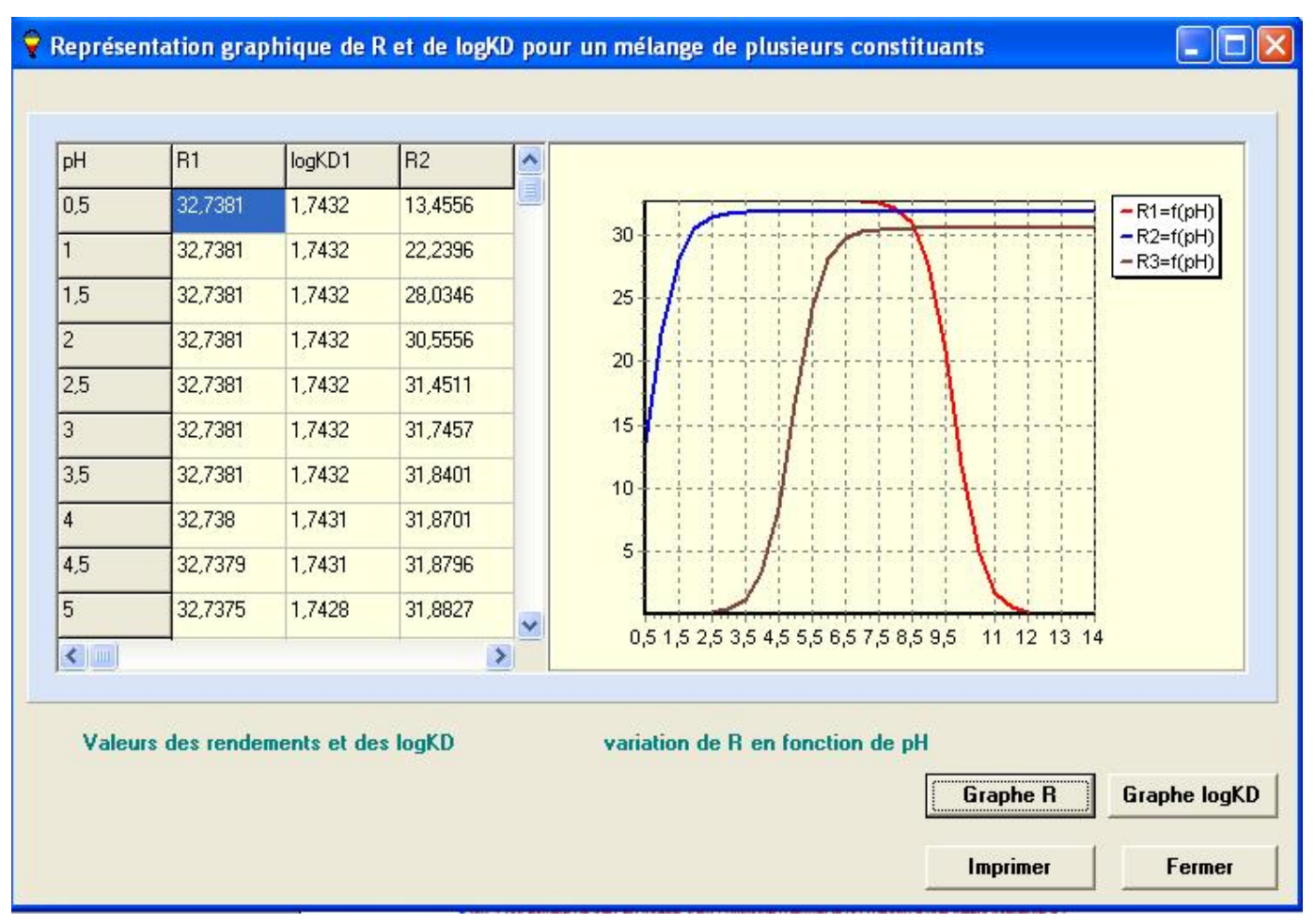

Fig.7. Variation du rendement en fonction du pH pour une extraction réactive de plusieurs constituants

\section{BASE DE DONNEES DES LOGK}

Le logiciel EXTRACT est doté d'une base de données contenant plus de 2000 valeurs des coefficients de partage $(\log K)$ pour 1000 substances environ, dans 36 solvants les plus usuels en extraction. Cette base de données offre l'avantage à l'utilisateur d'être modifiée ou enrichie par d'autres valeurs des $\log \mathrm{K}$ ou de nouveaux solvants, elle peut en contenir jusqu'à 100.000 valeurs.

\subsection{Recherche de $K$ dans la base de données}

Il faut cliquer sur le bouton Déterminer $\mathbf{K}$, dans l'une des feuilles de calcul du programme, une fiche intitulée «Recherche de K dans la base de donnée » est activée (voir figure ci dessous), il reste à sélectionner le solvant et la substance dans les menus contextuels et cliquer sur le bouton $\mathrm{OK}$, une fois la valeur de K est affichée, l'utilisateur peut l'emporter à l'endroit ou il veut l'utiliser après sélection de celle-ci et en validant par Ok. 


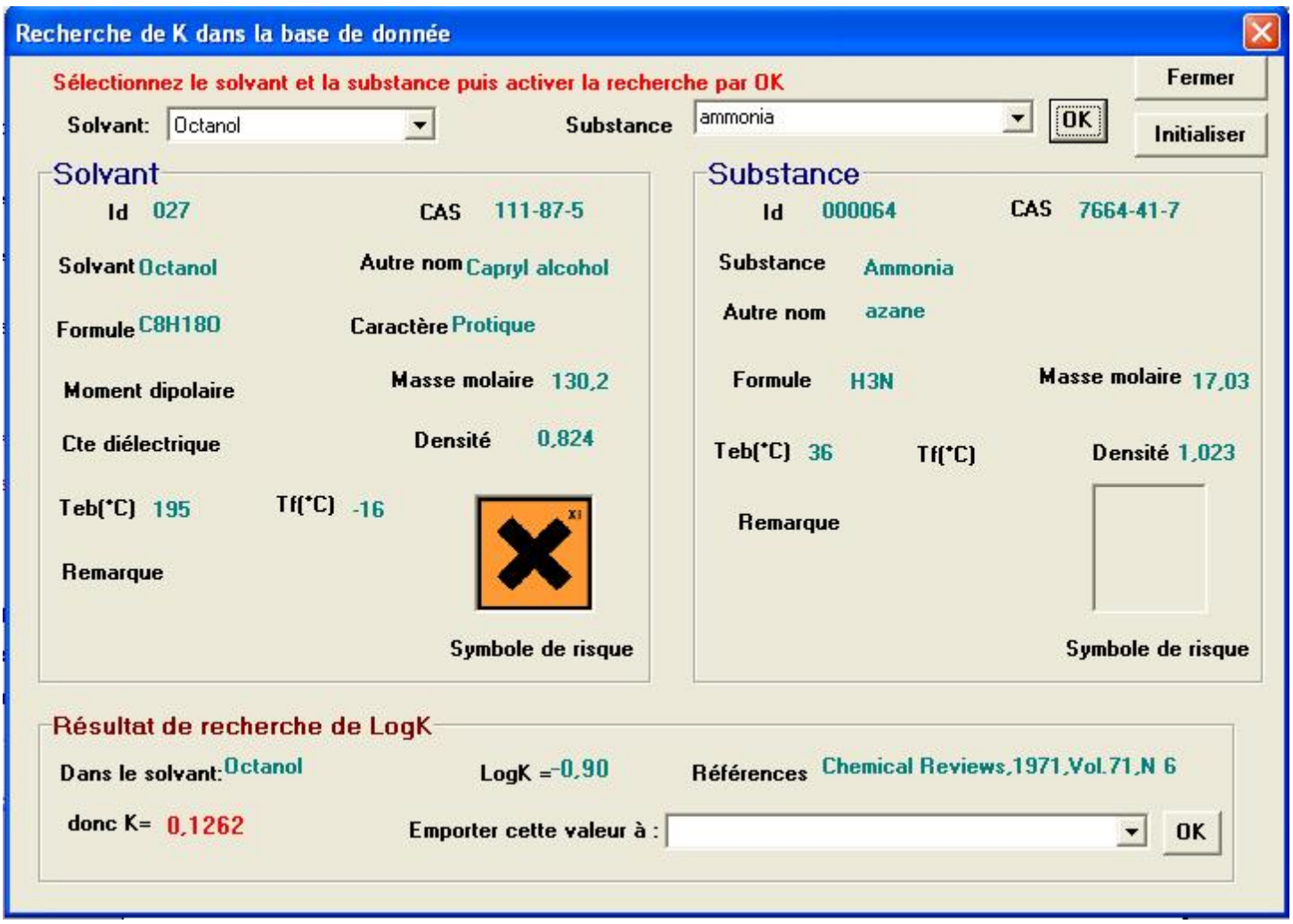

Fig.8. Recherche de K dans la base de données

\section{MENU D'AIDE DU LOGICIEL EXTRACT}

La rubrique d'aide de EXTRACT pourra être consultée en cas de besoin en appuyant sur Aide (figure 9). 


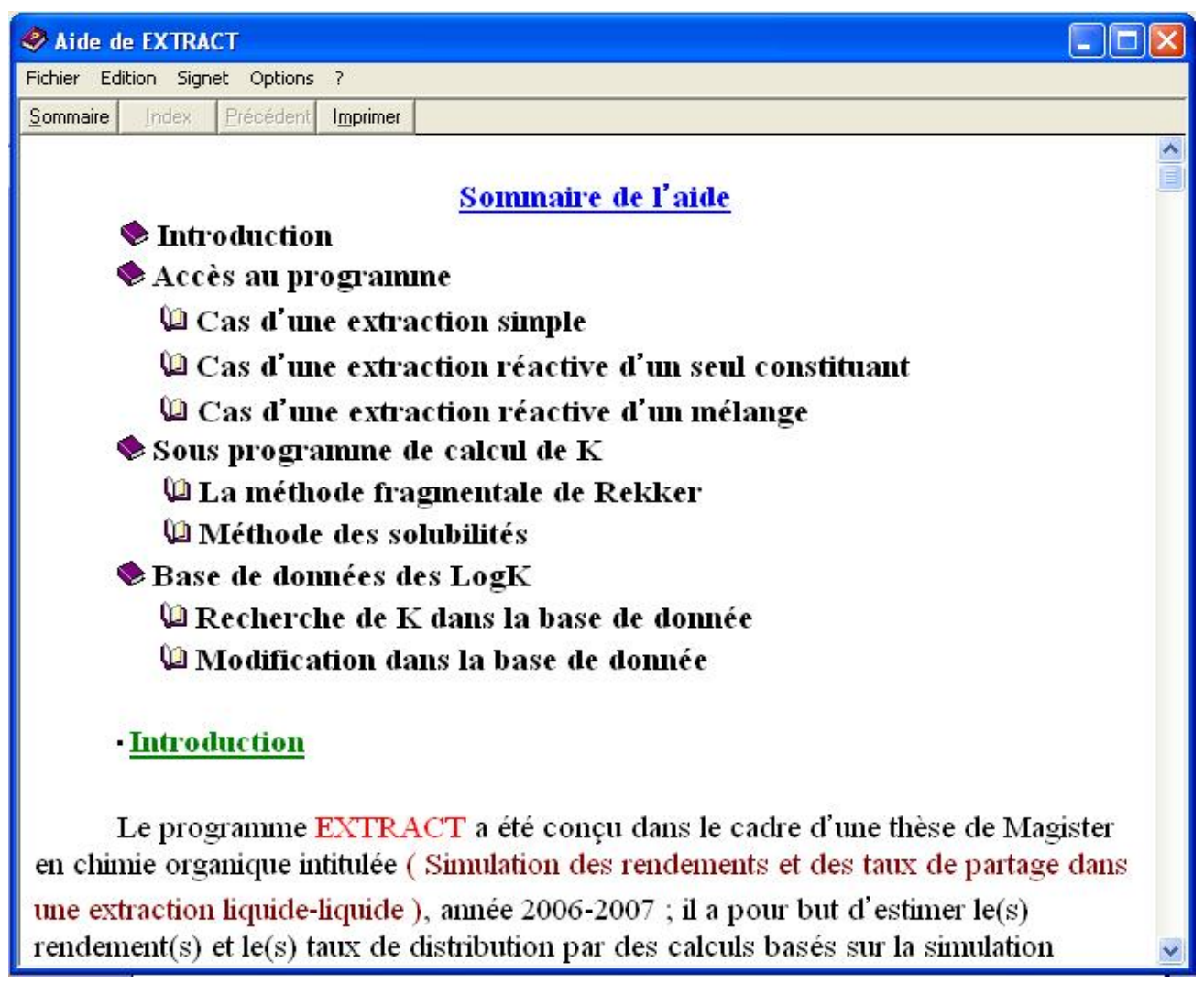

Fig.9. Rubriques d'aide de EXTRACT

\section{VALIDATION EXPERIMENTALE DU LOGICIEL}

Une validation expérimentale des résultats compilés par le logiciel a été appliquée à l'extraction d'un acide carboxylique (acide formique $\mathrm{HCOOH} 0,1 \mathrm{~N}$ ) en solution aqueuse par un solvant organique (butan-1-ol). Trois cas ont été mis en évidence: i) cas ou l'acide est le seul constituant du mélange; ii) l'acide formique est en présence d'un autre acide carboxylique (l'acide benzoïque $\mathrm{C}_{6} \mathrm{H}_{5} \mathrm{COOH} 0,025 \mathrm{~N}$ ) ; iii) l'acide est en mélange avec une base faible $\mathrm{NH}_{4} \mathrm{OH}$ 0,1N. Dans chaque cas, $10 \mathrm{ml}$ de solution aqueuse ont été agité par une quantité pareille de solvant, après séparation, la concentration de la phase aqueuse en acide a été déterminée par spectrophotométrie UV, celle de la phase organique par bilan, ainsi les valeurs du taux de partage et du rendement ont été déduites et comparés à ceux compilés par le logiciel.

Le tableau, ci-dessous, renferme les valeurs des taux de partage et des rendements de l'extraction de l'acide pour les trois cas étudiés. Il est évidemment remarquable que les résultats prédits sont très proches des valeurs expérimentales. 
Tableau 1. Valeurs du taux de partage et du rendement pour l'extraction de l'acide formique contenu seul en solution puis en mélange sans correction de $\mathrm{pH}$ (solvant: butan-1-ol; $\mathrm{V}_{\mathrm{aq}} / \mathrm{V}_{\text {org }}=1: 1 ; \mathrm{T}=20^{\circ} \mathrm{C}$ )

\begin{tabular}{|c|c|c|c|c|c|c|c|}
\hline $\begin{array}{c}\text { Mélange de } \\
\text { départ }\end{array}$ & $\begin{array}{c}\text { Entité } \\
\text { extraite }\end{array}$ & $\begin{array}{c}\mathbf{C} \text { initiale } \\
(\mathbf{m o l} / \mathbf{l})\end{array}$ & $\begin{array}{c}\mathbf{p H}_{\text {ex }} \\
\mathbf{p}\end{array}$ & $\begin{array}{c}\text { Taux part. D } \\
\text { cal }\end{array}$ & $\begin{array}{c}\text { Taux part. D } \\
\text { exp }\end{array}$ & $\begin{array}{c}\text { Rend. } \\
\mathbf{R}_{\text {cal }}(\%)\end{array}$ & $\begin{array}{c}\text { Rend. } \\
\mathbf{R}_{\text {exp }}(\%)\end{array}$ \\
\hline un acide & $\mathrm{HCOOH}$ & 0,1 & 2,29 & 0,8717 & 0,8571 & 46,5715 & 46,1523 \\
\hline \multirow{2}{*}{$\begin{array}{c}\text { Deux } \\
\text { acides }\end{array}$} & $\mathrm{HCOOH}$ & 0,1 & 2,47 & 0,8735 & 0,8727 & 37,2992 & 37,2800 \\
\cline { 2 - 8 } & $\mathrm{C}_{6} \mathrm{H}_{5} \mathrm{COOH}$ & 0,025 & - & 65,9032 & 61,5 & 19,7011 & 19,6800 \\
\hline \multirow{2}{*}{$\begin{array}{c}\text { Un aide et } \\
\text { une base }\end{array}$} & $\mathrm{HCOOH}$ & 0,1 & 5,01 & 0,0604 & 0,0721 & 2,8480 & 3,3626 \\
\cline { 2 - 8 } & $\mathrm{NH}_{4} \mathrm{OH}$ & 0,1 & - & 0,0011 & 0,0017 & 0,0549 & 0,0849 \\
\hline
\end{tabular}

\section{CONCLUSION}

La modélisation et la conception par ordinateur des équations d'équilibre liquideliquide d'un système multi-constituants nous a permet à travers le logiciel EXTRACT développé à l'aide de l'environnement orienté objet DELPHI, d'optimiser le processus d'extraction, le taux de partage et le rendement de l'opération ont été simulé et calculé avec une bonne approche en les comparant aux valeurs expérimentales.

Les résultats du logiciel pour le cas d'extraction de l'acide formique contenu seul en solution puis en mélange sont très cohérents à ceux déterminés expérimentalement, ce qui confirme bien l'authenticité et la validité du logiciel développé.

\section{REFERENCES}

[1] Lininger A., Jain A. Liquid-liquid extraction, Che 396 Senior Design.

[2] Sekine T., Hasegawa Y. 1977, Solvent Extraction Chemistry: Fundamentals and Application, Marcel Dekker, INC. New York and Basel.

[3] Wang Y., Li Y., Li Y., Wang J., Li Z., Dai Y. Extraction Equilibria of Monocarboxylic Acids with Trialkyl Phosphine Oxide, J Chem Eng Data. 2001, 6, 831837.

[4] Mahfud F. H., Van Geel F. P., Venderbosch R. H., Heeres H. J. Acetic Acid Recovery from Fast Pyrolysis Oil. An Exploratory Study on Liquid-Liquid Reactive Extraction Using Aliphatic Teriary Amines, Separation Science and Technology. 2008, 43, 3056-3074. 
[5] Salhi R., Bouhidel K. A Rigourous Calculation Method for Determining PotentialpH Diagrams in Metal-Ammonia-Water Systems, Asian Journal of Chemestry. 2005, 17(1), 245-258.

[6] Kyuchoukov G., Yankov D., Marinova M., Molinier J. and Albet J. Mechanism of lactic acid extraction with quaternary ammonium chloride (Aliquat 336), Ind. Eng. Chem. Res. 2005, 44, 5733-5739.

[7] Kertes A. S. and King C. J. Extraction chemistry of fermentation product carboxylic acids, Biotechnol. Bioeng. 1986, 28, 269-282.

[8] Chang-Hoon S. et al. A Solvent Extraction Approach to Recover Acetic Acid From Mixed Waste Acids Produced During Semicondutor Wafer Process, Journal of Hazardous Materials. 2009, 162, 1278-1284.

[9] Amit K., Shri C. and Kailas L. Recovery of Propionic Acid from Aqueous Phase by Reactive Extraction using Quarternary Amine (Aliquat 336) in Various Diluents, Chemical Engineering Journal. 2009, 152, 95-102.

[10] Fan M., Qun W., Dai Y. Extraction Equilibria of Benzoic Acid with Tributyl Phosphate in Kerosene and 1-Octanol, J. Chem. Eng. Data. 2002, 47, 941-943.

[11] Thomas B. 2002, Delphi 6, Campus Press, France, 07-11p.

[12] Belaid M. Juillet 2006, Programmer les bases de données Delphi, édition pages blues internationales, 13-22p. 


\section{CONTRIBUTION A L'ELABORATION D'UN LOGICIEL DE SIMULATION \\ DU RENDEMENT ET DU TAUX DE PARTAGE DANS UNE EXTRACTION \\ LIQUIDE-LIQUIDE}

\section{RESUME}

Le présent travail consiste à élaborer un logiciel permettant de prédire la valeur du rendement et du taux de partage dans le processus d'extraction liquide-liquide des constituants d'un mélange, à partir de modèles mathématiques exprimant ces entités, basés sur des équations d'équilibre liquide-liquide entre phases, et de prévoir dans quelles conditions l'opération d'extraction est favorable, défavorable ou impossible en étudiant la variation des entités susmentionnées en fonction des paramètres influençant sur l'extraction, à savoir, concentrations initiales, taux de solvant et $\mathrm{pH}$, dans le cas d'une extraction simple (extraction de produits neutres) ou lorsque celle-ci est réactive (extraction de complexes, acides ou bases), pour un ou plusieurs constituants.

Le langage de programmation utilisé est "Delphi" qui est un outil très puissant de programmation orientée objet sous Windows.

Mots clés: Extraction liquide-liquide, partage, rendement, coefficient de partage, taux de partage, solvant, soluté, Delphi.

\section{How to cite this article}

Hadj Seyd A, Lanez T. Contribution to the development of a simulation software performance and sharing ratio in liquid-liquid extraction. J Fundam Appl Sci. 2011, $3(2), 169-182$. 